\title{
How many signals does it take?
}

\section{T. V. Venkatesh and Rolf Bodmer}

\section{Summary}

Although the genetics of dorsal-ventral polarity which leads to mesoderm formation in Drosophila are understood in considerable detail, subsequent molecular mechanisms involved in patterning the mesoderm primordium into individual mesodermal subtypes are poorly understood. Two papers published recently ${ }^{(1,2)}$ suggest strongly that an inductive signal from dorsal ectoderm is involved in subdividing the underlying mesoderm, and present evidence that one of the signalling factors is Decapentaplegic (Dpp), a member of the bone morphogenetic protein subgroup of the Transforming Growth Factor- $\beta$ (TGF- $\beta$ ) super family of proteins.

\section{Introduction}

Specification of cell fate during development is a multistep process. Cells often receive different signals from neighboring cells at different stages during their development, which play a significant role in determining their developmental fate. Members of the TGF- $\beta$ superfamily, which are widely conserved in different organisms, constitute a major group of signalling molecules. They appear to mediate key events in normal growth and development and exhibit diverse activities like specification of body axis and induction of mesoderm in Xenopus, and control sexual development and creation of bones and cartilage in mammals (reviewed in ref. 4). In Drosophila, decapentaplegic (dpp) is required for formation of the embryonic dorsoventral axis of ectodermal cuticular structures, communication between tissue layers in gut development and correct proximal distal axis of adult appendages ${ }^{(5-9)}$. The two papers reviewed here demonstrate a new inductive role for $d p p$ expressed in the dorsal ectoderm, to pattern the underlying mesoderm during early embryogenesis ${ }^{(1,2)}$.

\section{Mesoderm formation in Drosophila}

Over the past two decades, enormous progress has been made in understanding the molecular mechanisms of cell fate specification in Drosophila. Most of these discoveries have concerned the specification of body axis, segmental patterning of the ectoderm and diversification in nervous system. Not much has been known about the molecular basis of mesodermal pattern formation and specification of mesodermal derivatives, with the exception of initial determination of the mesodermal anlagen. Mesoderm formation at blastoderm stage is controlled by a cascade of maternally active genes that generate dorso-ventral polarity. This process cul- minates in a nuclear gradient of the maternally expressed morphogen, dorsal, which determines the domains of two zygotic genes twist ( $t w i)$ and snail (sna) in the ventral region of the early embryo (for a review, see ref. 3). During gastrulation this region of the embryo invaginates along the ventral midline into the interior of the embryo to form the mesoderm (Fig. 1A). The products of these two genes are likely to act as transcription factors, and the activities of both are required for mesoderm formation. It has been suggested that twist, which encodes a basic helix-loop-helix protein, functions as a positive activator of mesoderm differentiation whereas snail, encoding a zinc finger protein, represses non-mesodermal genes in the mesodermal primordium. Mesodermal cells do not appear to be committed to particular developmental fates during and shortly after gastrulation(10) and additional factors are obviously involved in further differentiation of the mesoderm into individual tissue types. The molecular basis of events after the formation of mesodermal primordium is beginning to be understood.

Mesodermal subdivision apparently occurs after the completion of gastrulation. Following invagination, the mesodermal cell mass flattens into a single layer which migrates below the ectoderm dorsally and extends to the border between dorsal ectoderm and amnioserosa(11) (Fig. 1B). Shortly thereafter the first morphological manifestation of mesodermal subdivision is the formation of two layers in the dorsal mesoderm: the inner layer, the visceral mesoderm, located dorsally and interiorly, contributes to the visceral gut muscles, while the outer layer, which remains in contact with the ectoderm (and extends to ventral midline), gives rise to somatic body wall muscles ${ }^{(12)}$ (Fig. 1C). The presumptive cardiac mesoderm (heart precursors) derives from two rows of dorsal-most mesodermal cells on both sides of the embryo ${ }^{(13,14)}$ (Fig. 1C). Recently several genes 
with a function in the mesoderm have been isolated and their functional analysis has facilitated the study of the molecular basis of mesoderm differentiation (reviewed in refs 15 and 16). Two homeobox genes, tinman (tin) and bagpipe (bap) are required for determination of cell fates in the dorsal mesoderm ${ }^{(13,17,18)}$. tin, which becomes restricted to the dorsal mesoderm, specifies both visceral and cardiac mesoderm, whereas bap is involved in the formation of the visceral mesoderm only. tin specifies visceral mesoderm by controlling the expression of bap in the dorsal meso$\operatorname{derm}^{(18)}$.

\section{Mesoderm patterning: signalling from ectoderm by $d p p$}

$d p p$ is expressed throughout the dorsal half of the early embryo and is required for the development of dorsal and lateral derivatives of the epidermis $(5,6)$. Midway through embryogenesis, $d p p$ is also expressed in the visceral mesoderm and acts as an inductive signal across germlayers. $d p p$ controls midgut morphogenesis by inducing the expression of the homeotic gene labial in the underlying midgut endoderm (reviewed in ref. 7). TGF- $\beta$ proteins have also been shown to induce mesoderm in amphibian embryos (for a review, see ref. 4). Different members of the TGF- $\beta$ family can induce different mesodermal tissues in animal cap assays (reviewed in ref. 19). Earlier transplantation experiments in other insects had indicated a role for ectoderm in patterning the mesoderm $(20,21)$. More recently an instructive role for the ectoderm in mesoderm patterning was suggested by experiments involving a block of gastrulation movements ${ }^{(22)}$. Experiments described by StaehlingHampton et al. (1) and Frasch ${ }^{(2)}$ in the two papers discussed here address the role of Dpp as an inductive signal secreted by the ectoderm in patterning the mesoderm. By ectopically expressing $d p p$ in the ventral ectoderm or in the mesoderm itself, they show that Dpp acts as an inductive signal from the dorsal ectoderm to regulate tin and bap expression in the underlying dorsal mesoderm. As a consequence, the mesoderm subdivides into somatic, visceral and cardiac mesoderm.

Although tin is initially expressed uniformly in the mesoderm and requires the function of $t w x^{(13)}$ (T. V. Venkatesh and $\mathrm{R}$. Bodmer, unpublished), shortly after mesodermal migration tin expression is diminished in the ventral portions of the mesoderm but is maintained in the dorsal mesoderm ${ }^{(17,18)}$. Later tin expression splits into two domains, the visceral mesoderm (transient expression) and the precursor cells of the heart (persistent expression), but is excluded from the somatic mesoderm. bap is first expressed in the dorsal mesoderm in segmental patches of cells that migrate inside to give rise to visceral mesoderm(18).

By analyzing the expression of tin and $d p p$ in the same embryo, Frasch(2) has shown that mesodermal cells expressing tin in the dorsal region of the mesoderm are located directly below the dorsal ectodermal cells expressing dpp. Mesodermal cells that do not 'contact' dpp lose tin mRNA expression shortly after dorsal migration of the mesoderm. Expression of $d p p$ was monitored with a $L a c Z$ reporter gene driven by $d p p$ regulatory sequences and tin was detected by mRNA expression. In contrast to wild-type, in $d p p$ mutant embryos tin expression is not maintained in the dorsal mesodermal cells. However, the early twi-dependent expression of tin in all the mesoderm was not affected in $d p p$ mutants. This loss of tin expression resulted in the absence of bap expression, and in turn no visceral mesoderm formed. Another consequence of loss of tin expression in the dorsal mesoderm was the failure to develop cardiac mesoderm. These results suggest that Dpp may act as a signalling molecule secreted from the ectoderm, required for maintaining tin expression in the dorsal mesoderm and for the formation of visceral and cardiac mesoderm. To test this hypothesis, $d p p$ was expressed in the entire dorsoventral circumference of the ectoderm but not in the mesoderm. This was achieved by using the GAL4 targeting system ${ }^{(23)}$. This is a powerful system for specifically targeting the expression of cloned genes in desired tissues in Drosophila. In this method, transgenic flies carry-
Fig. 1. Gastrulation and mesoderm formation in Drosophila. (A-C) Schematic representation of transverse sections of Drosophila embryos at different stages. Embryos are oriented dorsal surface up. (A) Blastoderm stage embryo ( 3 hours of development) showing the fate of cells along the dorsoventral axis. Ventral-most cells (red ovals) are the primordial mesodermal cells that during gastrulation ( 3.5 hours of development) invaginate (see text). (B) Early gastrula (4.5 hours of development). The invaginated mesoderm has spread dorsally along the basal surface of the ectoderm. Mesoderm cells juxtaposed to the dorsal ectoderm are induced by dpp to maintain tin expression (arrows), while ventral cells (not induced) lose tin expression. (C) Embryos after subdivision of the mesoderm: dorsal mesodermal cells differentiate into different tissue types. Additional signals (?) from ectoderm (arrows) are probably required for differentiation of the cardiac mesoderm.

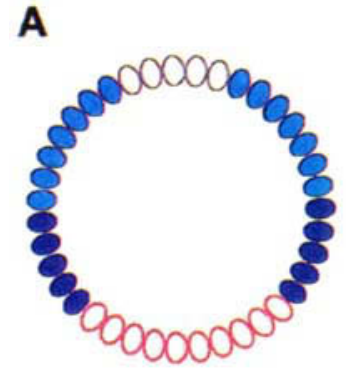

B

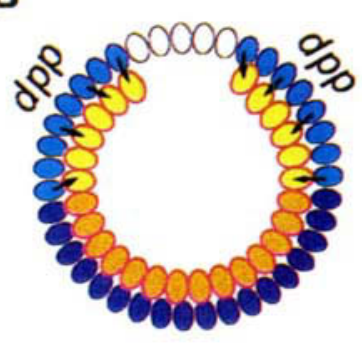

C

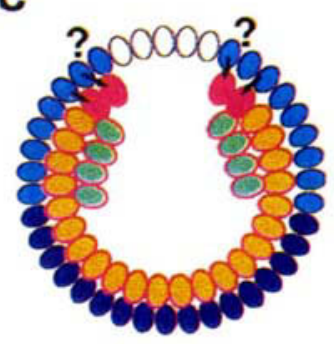

cardiac mesoderm

visceral mesoderm somatic mesoderm mesoderm

ventral mesoderm

dorsal mesoderm ventro-lateral ectoderm amnioserosa 
ing GAL4, a potent transcriptional activator, from yeast driven by a tissue-specific enhancer of choice are crossed to another transgenic line, carrying the cDNA of the gene of interest (in this case $d p p$ ) under the control of GAL4 upstream activating sequences (UAS). To obtain ectopic expression of $d p p$ in the ventral mesoderm, Frasch ${ }^{(2)}$ used a synthetic enhancer which combined the enhancer elements from the gap gene kruppel and ventral repressor elements of zen ${ }^{(24)}$ to drive GAL4 in the ectodermal primordium, but not in the mesodermal anlagen. As expected of a necessary and sufficient inductive signal for specifying dorsal mesoderm, ectopic expression of $d p p$ in all ectodermal cells caused an expansion of tin expression towards the ventral midline. The ventrally maintained tin expression resulted in a similar expansion of the bap domain and the visceral mesoderm towards ventral parts of the mesoderm. These observations put $d p p$ in a strong position as an inductive signal for mesodermal patterning. Surprisingly expansion of dorsal mesoderm did not cause a proportionate expansion of the cardiac mesoderm: the number and location of the heart progenitors appeared unaltered. Thus, additional patterning factors must contribute to the spatial specification of the heart.

Staehiling-Hampton et al.(1) used a similar approach to ectopically express $d p p$ with the GAL4 system. In contrast to the other paper, they expressed $d p p$ throughout the mesoderm, to test whether or not $d p p$ can induce dorsal mesoderm when it is expressed in the ventral mesodermal primordia. This was achieved by using the enhancer elements of twi to drive GAL4 expression. In embryos carrying twi-GAL4 and UAS-dpp, bap expression appeared prematurely as mesodermal cells invaginated through the ventral furrow and its domain remained expanded to include the ventral mesoderm, similar to Frasch's observations ${ }^{(2)}$. Thus, supplying of Dpp directly by the mesoderm is sufficient to induce dorsal mesoderm. As tin is present uniformly throughout the mesoderm at this stage, this result suggests that Dpp does not simply act through tin for bap expression in the mesoderm, but in addition seems to be required in parallel to tin. Therefore, it is possible that Dpp-dependent factors may be able to directly activate or maintain bap expression along with tin in the dorsal mesoderm of the wildtype embryo. To address the question of whether or not induction of dorsal mesoderm suppresses ventral mesoderm, Staehling-Hampton et al.(1) also expressed $d p p$ throughout the ectoderm by an ectoderm-specific GAL4 line, $69 \mathrm{~B}^{(23)}$. In these embryos bap expression extended ventrally (see also Frasch(2)), while the expression of poxmeso, a transcription factor normally expressed in the ventral mesoderm, was absent. Therefore $d p p$ is not only involved in the activation of dorsally restricted mesodermal genes, but may also direct the repression of genes whose expression is normally confined to the ventral mesoderm. Consistent with this conclusion, in embryos mutant for $d p p$, not only was a loss of bap expression in the dorsally located mesoderm observed, but also expanded expression of poxmeso throughout the circumference of the embryo. Interestingly, the results further show that expression of $d p p$ in the mesoderm can generate completely dorsalized cuticle, implying that Dpp can act across germlayers in either direction.

\section{Implications and future studies}

These studies clearly demonstrate a role for Dpp in patterning the mesoderm: Dpp affects the differentiation of the dorsal mesoderm by controlling the expression of mesodermal genes. Considering that Dpp is a secretory signalling molecule, there should be a distinct signalling pathway involved in this cellular communication. Recently serine/ threonine transmembrane kinase receptors for Dpp have been identified ${ }^{(25,26)}$ (for a review, see ref. 27). One of the type I receptors encoded by thickvein is expressed in the mesoderm ${ }^{(26)}$. Other molecules involved in transducing the Dpp signals downstream of these receptors have not been identified, with the exception of schnurri, a recently identified zinc-finger-containing putative transcription factor gene. Apparently, the schnurri transcription factor mediates Dpp signalling from the visceral mesoderm to the adjacent endoderm ${ }^{(28,29)}$ and presumably also in the early mesoderm ${ }^{(28)}$. It would be interesting to see if schnurri directly controls the expression of tin and bap by binding to their promoters.

It is not known what else contributes to the determination of the spatial domain of cardiac mesoderm. There should be additional factors which mediate the patterning of the cardiac mesoderm (see ?, Fig. 1C), since ectopic expression of the $d p p$ in the ectoderm did not alter the domain of heart progenitor cells. Interestingly, very recently it has been reported that the secreted signalling molecule, Wingless $(\mathrm{Wg})$, which is known for its function in determining the anterior-posterior segment-polarity of the ectoderm (reviewed in ref. 30 ), is involved in the formation of cardiac mesoderm in Drosophila $^{(31)}$. By using a temperature-sensitive allele of $w g$, it has been shown that elimination of $w g$ gene function for a short time period after gastrulation results in the selective elimination of the heart progenitor cells. Formation of the visceral mesoderm and segmentation was essentially normal in these embryos. Elimination of $w g$ function resulted in the selective loss of tin expression in the presumptive cardiac precursor cells, without affecting the tin expression in the early mesoderm or its subsequent dorsal restriction. Further experiments show that $\mathrm{Wg}$ may be a direct signal for heart formation as overexpression of $w g$ rescues heart formation in mutants of hedgehog (M. Park, X. Wu, K. Golden and R. Bodmer, unpublished). It thus appears that both dorso-ventral and anterior-posterior patterning signals from ectoderm are involved in patterning the mesoderm. Taken together these studies indicate that the mesoderm development involves complex molecular mech- 
anisms that are mediated by signals from the ectoderm and factors endogenous to the mesoderm. Given the fact that many mesodermal genes including tinman, Dmef2 and nautilus (myoD homologue) are conserved in vertebrates, it will be interesting to see how these recent discoveries in Drosophila lead to new discoveries in vertebrate mesoderm patterning.

\section{References}

1 Staehling-Hampton, K., Hoffmann, F.M., Baylies, M.K., Rushton, E. and Bate, M. (1994). dpp induces mesodermal gene expression in Drosophila. Nature 372, 783-786

2 Frasch, M. (1995). Induction of visceral and cardiac mesoderm by ectodermal Dpp in the early Drosophila embryo. Nature 374, 464-467.

3 Govind, S. and Steward, R. (1991). Dorsoventral pattern formation in Drosophila. Trends Genet. 7, 119-125.

4 Kingsley, D. (1994). The TGF- $\beta$ superfamily: new members, new receptors, and new genetic tests of function in different organisms. Genes Dev. 8, 133-146.

5 Spencer, F.A., Hotfmann, F.M. and Gelbert, W.M. (1982). decapentaplegic: A gene complex affecting morphogenesis in Drosophila melanogaster. Cell 28, 451 461.

6 Ferguson, E.L. and Anderson, K.V. (1992). decapentaplegic acts as a morphogen to organize dorsal-ventral pattern in the Drosophila embryo. Cell 71 , 451-461.

7 Bienz, M. (1994). Homeotic genes and positional signalling in the Drosophila viscera. Trends Genet. 10, 22-26.

8 Perrimon, N.(1995). Hedgehog and beyond. Cell 80, 517-520.

9 Blair, S.S. (1995). Compartments and appendage development in Drosophila. BioEssays 17, 299-309.

10 Beer, J., Technau, G. and Campos-Ortega, J.A. (1987). Lineage analysis of transplanted individual cells in embryos of Drosophila melanogaster. IV. Commitment and proliferative capabilities of mesodermal cells. Roux's Arch. Dev. Biol. 196, 222-230.

11 Leptin, M. and Grunewald, B. (1990). Cell shape changes during gastrulation in Drosophila. Development 110, 73-84.

12 Bate, M. (1993). The mesoderm and derivatives. The Developmental Biology 2, 1013-1090.

13 Bodmer, R., Jan, L.Y. and Jan, Y.N. (1990). A new homeobox-containing gene, $m s h-2$ (tinman), is transiently expressed early during mesoderm formation in Drosophila. Development 110, 661-669.

14 Rugendorff A., Younossi-Hartenstein, A. and Hartenstein, V. (1994). Embryonic origin and differentiation of the Drosophila heart. Roux's Arch Dev. Biol. 203, 266-280.
15 Bodmer, R. (1995). Heart development in Drosophila and its relationship to vertebrate systems. Trends Cardiovasc. Med. 5, 21-27.

16 Abmayr, S.M., Erickson, S.M. and Bour, B.A. (†995). Embryonic development of larval body musculature of Drosophila melanogaster. Trends Genet. 11, 153-159.

17 Bodmer, R. (1993). The gene tinman is required for specification of the heart and visceral muscles in Drosophila. Development 118, 719-729.

18 Azpiazu, N. and Frasch, M. (1993). tinman and bagpipe: two homeobox genes that determine cell fates in the dorsal mesoderm of Drosophila. Genes Dev. 7, $1325-1340$

19 Smith, J.C. and Howard, J.E. (1992). Mesoderm inducing factors and the control of gastrulation. Development (supplement), 127-136.

20 Kuhn, A. (1971). Lectures in Developmental Physiology. New York: SpringerVerlag.

21 Counce, S.J. (1973). The casual analysis of insect development. In Developmental Systems: Insects (vol. 2) (ed. S.J. Counce and C.H. Waddington), pp. 1-156, London: Academic Press.

22 Baker, R. and Schubiger, G. (1995). Ectoderm induces muscle-specific gene expression in Drosophila embryos. Development 121, 1387-1398.

23 Brand, A.H. and Perrimon, N. (1993). Targeted gene expression as a means of altering cell fates and generating dominant phenotypes. Development 118, 401 415.

24 Ip, Y.T., Kruat, R., Levine, M. and Rushlow, C.A. (1991). The dorsal morphogen is a sequence specific DNA binding protein that interacts with a sequence specific long range repression eiement. Cel/64, 439-446.

25 Ruberte, E., Marty, T., Nellen, D., Affolter, M. and Basler, K. (1995). An absolute requirement for both the type II and type I receptors, Punt and Thick veins, for Dpp signaling in vivo. Cell 80, 889-897.

26 Letsou, A. et al. (1995). Drosophila Dpp signaling is mediated by the punt gene product: A dual ligand-binding type II receptor of the TGF- $\beta$ receptor family. Cell 80, 899-908.

27 Wharton, K.A. (1995). How many receptors does it take? BioEssays 17, 13-16. 28 Arora, K., Dai, H., Kazuko, S.G., Jamal, J., O'Connor, M.B., Letsou, A. and Warrior, R. (1995). The Drosophila schnurrigene acts in the Dpp/TGF- $\beta$ signaling pathway and encodes a transcription factor homologous to the human MBP tamily. Cell 8, 781-790.

29 Grieder, N.C., Nellen, D., Burke, R., Basler, K. and Affolter, M. (1995) Schnurri is required for Drosophila Dpp signalling and encodes a zinc finger protein similar to mammalian transcription factor PRDII-BFI. Cell 81, 791-800. 30 Perrimon, N. (1994). The genetic basis of patterned baldness in Drosophila Cell 76, 781-784.

31 Wu, X., Golden, K. and Bodmer, R. (1995). Heart development in Drosophila requires the segment polarity gene wingless. Dev. Biol. 169, 619-628.

T. V. Venkatesh and Rolf Bodmer are at the Department of Biology, University of Michigan, Ann Arbor, MI-48109, USA. 